\title{
Resummation of Cactus Diagrams in Lattice QCD, to all Orders
}

\author{
H. Panagopoulos ${ }^{\mathrm{a}}$, E. Vicari ${ }^{\mathrm{b}}$

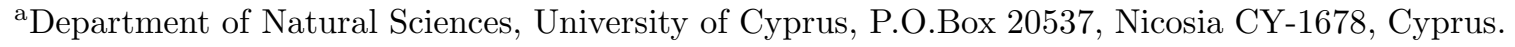 \\ ${ }^{\text {b}}$ Dipartimento di Fisica dell'Università and I.N.F.N., via Buonarroti 2, I-56127 Pisa, Italy.
}

We show how to perform a resummation, to all orders in perturbation theory, of a certain class of gauge invariant tadpole-like diagrams in Lattice QCD. These diagrams are often largely responsible for lattice artifacts. Our resummation leads to an improved perturbative expansion. Applied to a number of cases of interest, e.g. the lattice renormalization of some two-fermion operators, this expansion yields results remarkably close to corresponding nonperturbative estimates. We consider in our study both the Wilson and the clover action for fermions.

\section{INTRODUCTION}

Ever since the earliest days of lattice field theory, one problem present in most numerical simulations has been the calculation of corrections induced by renormalization on Monte Carlo results. Several methods have been used to address it. Perturbation theory provides in principle a methodical means of calculating renormalization functions, operator mixing coefficients, etc. Its drawbacks lie in its asymptotic nature, and in that it is a formidable task on the lattice, which places severe limitations on the order to which it can be carried out; indeed, at present, exact calculations in perturbative lattice QCD reach only two loops (for 2-point diagrams) 113] and three loops (for vacuum diagrams) [4. In recent years there have been considerable efforts in refining the perturbative computations introducing recipes motivated by mean field and tadpole resummation arguments [5]. Various nonperturbative, numerical approaches to renormalization functions have also been devised and there has been recent progress both in their range of applicability and in their precision 6 8]. Nonperturbative methods, such as those of Refs. 68] are in general preferable to approximations based on perturbative calculations, due to their better controlled systematic errors $(O(a)$ against $\left.O\left(g_{0}^{n}\right)\right)$. However, improved perturbative estimates are still quite useful. They indeed provide important consistency checks. Further, in those cases where nonperturbative methods are difficult to implement, perturbative methods remain the only source of quantitative information.

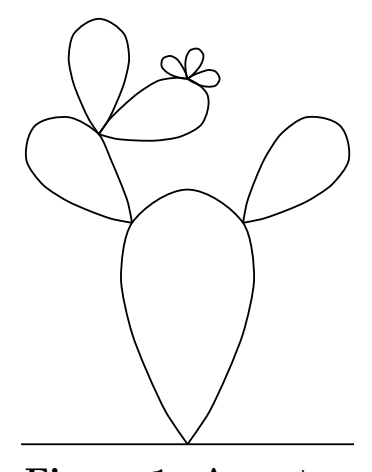

Figure 1: A cactus

We present an improvement of lattice perturbation theory, which results from a resummation to all orders of a certain class of diagrams, dubbed "cactus" diagrams [9.10. Briefly stated, these are tadpole diagrams which become disconnected if any one of their vertices is removed (see Figure 1). Our original motivation was the well known observation of "tadpole dominance" in lattice perturbation theory. This observation must clearly be taken with a grain of salt: One-sided inclusion of tadpoles can ruin desirable partial cancellations between tadpole and non-tadpole diagrams; worse, their contribution is gauge dependent. The class of terms we propose to resum circumvents the latter objection since, as we shall see, it is gauge invariant; it also overcomes the former objection in known cases. Given its diagram- 
matic nature, our method leads to results which can be systematically improved, order by order. Applied to a number of cases of interest, the cactus resummation yields remarkable improvements when compared with the available nonperturbative estimates. As regards numerical comparison with other improvement schemes, such as boosted perturbation theory [5], our method fares equally well on all the cases studied.

\section{THE CALCULATION}

Consider the standard Wilson action for $S U(N)$ lattice gauge fields:

$$
S=\frac{1}{g_{0}^{2}} \sum_{x, \mu \nu} \operatorname{Re} \operatorname{tr}\left(1-U_{x, \mu \nu}^{\square}\right)
$$

$U_{x, \mu \nu}^{\square}$ is the usual product of link variables around a plaquette in the $\mu-\nu$ plane with origin at $x$; in standard notation:

$$
U_{x, \mu \nu}^{\square}=e^{i g_{0} A_{x, \mu}} e^{i g_{0} A_{x+\mu, \nu}} e^{-i g_{0} A_{x+\nu, \mu}} e^{-i g_{0} A_{x, \nu},}
$$

where $A_{x, \mu}=A_{x, \mu}^{a} T^{a}$. By the Baker-CampbellHausdorff $(\mathrm{BCH})$ formula we have:

$U_{x, \mu \nu}^{\square}=\exp \left\{i g_{0} F_{x, \mu \nu}^{(1)}+\mathcal{O}\left(g_{0}^{2}\right)\right\}$

$F_{x, \mu \nu}^{(1)} \equiv A_{x, \mu}+A_{x+\mu, \nu}-A_{x+\nu, \mu}-A_{x, \nu}$

The diagrams which we propose to resum to all orders will be cactus diagrams made of vertices containing $F_{x, \mu \nu}^{(1)}$. Let us see how such diagrams will dress the gluon propagator; we write:

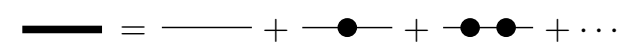

where the one-particle irreducible piece is given by the recursive equation:

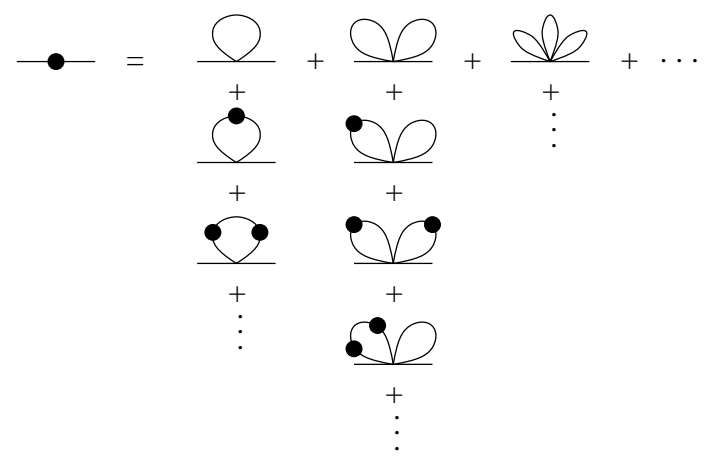

The presence of only $F_{x, \mu \nu}^{(1)}$ in the vertices ensures that the longitudinal parts of all propagators cancel, so that the effect of dressing is the same in all covariant gauges. Thus, we can write:

$$
\longrightarrow=w\left(g_{0}\right) \cdot
$$

Indeed, the dressed propagator will become a multiple of the bare transverse one, where the factor $w\left(g_{0}\right)$ will depend on $g_{0}$ and $N$, but not on the momentum. The above diagrammatic relations can be turned into an algebraic equation for $w\left(g_{0}\right)$ that can be easily solved numerically.

Similar to the dressing of the propagator, one dresses the action vertices, operator insertions, etc. Moreover these calculations can be extended [10] to the case of the clover improved action formulation of lattice QCD [11], which is widely used in numerical simulations in order to reduce scaling corrections.

Cactus resummation may be applied either to bare quantities or to quantities which have been calculated to a given order in perturbation theory; thus contributions which are not included in the resummation can be reintroduced in a systematic manner.

\section{APPLICATIONS OF CACTUS RE- SUMMATION}

The resummation of the cactus diagrams can be applied to the calculation of the renormalization of some lattice operators. Approximate expressions of the lattice renormalizations are obtained by dressing the corresponding one-loop calculations.

As first example [9] we consider the renormalization $Z\left(g_{0}\right)$ of the topological charge density lattice operator

$Q(x)=-\frac{1}{2^{4} \times 32 \pi^{2}} \sum_{\mu, \nu, \rho, \sigma= \pm 1}^{ \pm 4} \varepsilon^{\mu \nu \rho \sigma} \operatorname{tr}\left\{U_{x, \mu \nu} U_{x, \rho \sigma}\right\}$

using the Wilson action and for $S U(3)$. $Z\left(g_{0}\right)$ is a finite function of $g_{0}$, going to one in the limit $g_{0} \rightarrow 0$, and that is much smaller than one in the region $g_{0} \simeq 1$, where Monte Carlo simulations using the Wilson action are actually performed. In perturbation theory one has 12 
$Z\left(g_{0}\right)=1-0.908 g_{0}^{2}+O\left(g_{0}^{4}\right)$, leading to the estimate $Z\left(g_{0}=1\right) \simeq 0.092$. The large one-loop coefficient suggests already that perturbation theory can hardly provide an acceptable estimate of $Z\left(g_{0}\right)$ for $g_{0} \simeq 1$ without some kind of resummation. Indeed the heating-method [13], which does not rely on perturbation theory, gives the estimate $Z\left(g_{0}=1\right)=0.19(1)$. Cactus dressing of the one-loop expression leads to

$$
\left.Z_{(} g_{0}^{2}\right) \approx\left[1-w\left(g_{0}\right)\right] \times\left[1-w\left(g_{0}\right)-0.491 g_{0}^{2}\right]
$$

Since $w\left(g_{0}=1\right) \simeq 0.250$, one finds $Z\left(g_{0}=1\right) \simeq$ 0.193 , in agreement with the heating-method result. A similar estimate is obtained by "meanfield" improving [5 the one-loop calculation: $Z \simeq$ $P^{2}\left(1-0.241 g_{P}^{2}\right)=0.209$, where $P \simeq 0.5937$ is the plaquette and $g_{P}^{2}=g_{0}^{2} / P$ a new coupling. Further confirmation [9] of the validity of the cactus resummation has been achieved for $S U(2)$.

One can also apply cactus resummation to the lattice renormalization of fermionic operators. In particular we consider the renormalizations $Z_{V}, Z_{A}$ of isovector fermionic currents using the clover action 10. Their one-loop result is known [14], $Z_{V, A}=1+g_{0}^{2} z_{V, A}\left(c_{\mathrm{SW}}\right)+\ldots$ Cactus dressing amounts to:

$$
1+\frac{g_{0}^{2}}{1-w\left(g_{0}\right)} z_{V, A}\left(\left(1-w\left(g_{0}\right)\right) c_{\mathrm{SW}}\right)+\ldots
$$
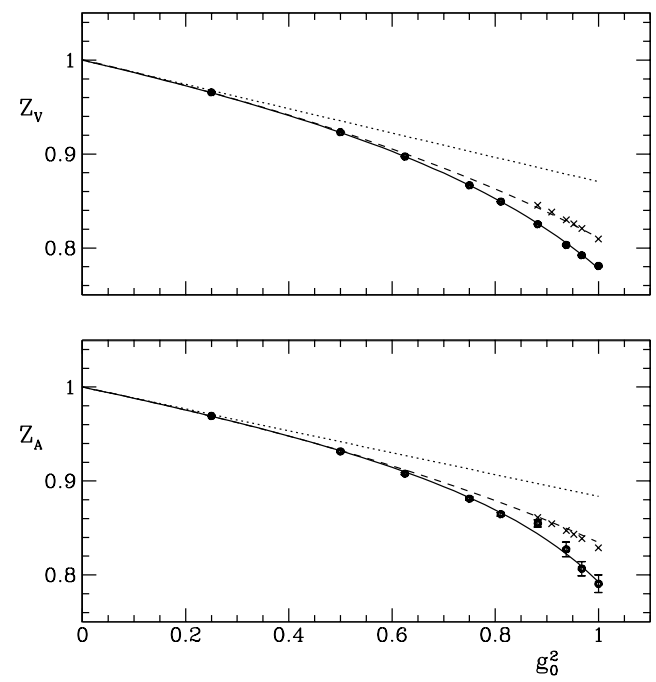

In the above figures we show results for $Z_{V}$ and $Z_{A}$ (from Ref. [8]), coming from numerical simulations (filled circles, fitted by a solid line), bare perturbation theory (dotted lines) and "mean field improved" perturbation theory [5] (crosses). The superimposed dashed lines are our results from cactus dressing. As this comparison shows, the dressed one-loop expressions constitute a remarkable improvement with respect to the simple one-loop calculation.

\section{REFERENCES}

1. M. Lüscher and P. Weisz, Nucl. Phys. B452, 234 (1995).

2. B. Allés, A. Feo, and H. Panagopoulos, Nucl. Phys. B491, 498 (1997).

3. C. Christou, A. Feo, H. Panagopoulos, and E. Vicari, Nucl. Phys. B525, 387 (1998).

4. B. Allés, A. Feo and H. Panagopoulos, Phys. Lett. B426, 361 (1998).

5. G. P. Lepage and P. B. Mackenzie, Phys. Rev. D48, 2250 (1993).

6. M. Bochicchio, L. Maiani, G. Martinelli, G. Rossi, and M. Testa, Nucl. Phys. B262, 331 (1985).

7. G. Martinelli, C. Pittori, C. T. Sachrajda, M. Testa, and A. Vladikas, Nucl. Phys. B445, 81 (1995).

8. M. Lüscher, S. Sint, R. Sommer and H. Wittig, Nucl. Phys. B491, 344 (1997).

9. H. Panagopoulos and E. Vicari, Phys. Rev. D58, 114501 (1998).

10. H. Panagopoulos and E. Vicari, Phys. Rev. D59, 057503 (1999).

11. B. Sheikholeslami and R. Wohlert, Nucl. Phys. B259, 572 (1985).

12. M. Campostrini, A. Di Giacomo, and H. Panagopoulos, Phys. Lett. B212, 206 (1988).

13. A. Di Giacomo and E. Vicari, Phys. Lett. B275, 429 (1992).

14. S. Capitani et al., Nucl. Phys. B (Proc. Suppl.) 63, 874 (1998). 